\title{
Paradoxical Size Effects In Composite Laminates And Other Heterogeneous Materials
}

\author{
M.A. Wheel \\ Department of Mechanical and Aerospace Engineering \\ University of Strathclyde \\ Glasgow, G1 1XJ, UK \\ marcus.wheel@strath.ac.uk \\ Tel +44 1415483307 \\ Fax +441415525105
}

\begin{abstract}
Size effects in which there is an apparent increase in stiffness with reducing size scale are forecast in those heterogeneous materials that have constitutive behaviour described by more generalized continuum theories such as couple stress, micropolar or micromorphic elasticity. This short paper considers possibly the simplest heterogeneous material exhibiting such size effects, a two phase composite laminate consisting of alternating layers of stiff and compliant material, and shows that when loaded in bending the nature of the size effect actually depends on the composition of the sample surfaces. The laminate material is apparently capable of exhibiting a diversity of size effects some of which are compatible with the predictions of generalized continuum theories while others are contradictory. Another heterogeneous material consisting of a periodic or regular array of voids within a classically elastic matrix is then considered. Detailed finite element analysis shows that the diversity of size effects encountered in the laminate material may also be observed in this more representative material thereby providing some insight into the contradictory size effects that have sometimes been reported elsewhere in the literature.
\end{abstract}




\section{Introduction}

Loaded materials are often assumed to deform in a manner described by classical or Cauchy elasticity theory which presumes that the material stiffness, quantified by its modulus, will be independent of size scale. The fact that many engineering materials actually demonstrate such size independent behaviour across those size scales of interest has resulted in the almost unanimous acceptance of this theory. However, there are some materials that do exhibit size dependent behaviour when loaded. Such behaviour has been observed in fabricated materials like foams [Lakes, 1983, 1986; Anderson \& Lakes, 1994] as well as biological tissues such as bone [Yang \& Lakes, 1982; Choi et al., 1990]. The size dependency apparently results from the size scale of their microstructure which may be sufficient to influence their macroscopic behaviour.

More generalized continuum theories with the capacity to forecast size dependent behaviour do exist and while some of these incorporate higher derivatives of the deformation into the constitutive equations others contain additional degrees of freedom. Examples of the latter type include in ascending level of complexity: couple stress, Cosserat or micropolar and micromorphic elasticity theories [Eringen, 1999]. Common to all of these theories is the incorporation of additional constitutive parameters that must be identified through experimentation on real materials or simulation of virtual materials. Parameter identification invariably involves testing or simulating material samples of different sizes in loading modes that induce a non uniform state of stress such as torsion or bending to reveal any size dependency [Lakes, 1995]. The observed size effect can then be used to identify the additional constitutive parameters. The generalized continuum theories already mentioned predict size effects in which size scale reduction results in an apparent increase in stiffness. Behaviour like this has been observed in both polymeric foams [Lakes, 1983, 1986; Anderson \& Lakes, 1994] and materials comprised of a two dimensional homogeneous matrix perforated by a regular array of circular voids [Beveridge et al., 2013; Waseem et al., 2013; McGregor \& Wheel, 2014]. However, there are materials such as cortical bone, for which both increasing and decreasing stiffness with reducing size have been reported [Yang \& Lakes, 1982; Choi et al., 1990]. This contradictory behaviour has been attributed to surface effects induced in sample preparation that increase compliance and result in a distorted size effect. Careful sample preparation has been highlighted as a requirement to avoid such distortion [Anderson \& Lakes, 1994]. While sample preparation may give rise to unanticipated size effects, the influence of the material microstructure on surface behaviour might also be a possible cause. Indeed, size effects in transversely loaded beams were recently [Gao \& Mahmoud, 2014] shown to depend on the combined behaviour of bulk and surface where these were represented by a generalized continuum theory and a surface elasticity model respectively.

It has also been reported that composite or laminate materials demonstrate behaviour consistent with the predictions of micropolar or Cosserat elasticity including the dispersion of propagating elastic waves when loaded dynamically [Herrmann \& Achenbach, 1968] and also a size dependent stiffness when loaded statically [Forest \& Sab, 1998]. This paper 
considers a simple laminate comprised of just two alternately layered constituents of differing moduli and demonstrates via a straightforward analysis that a rich variety of size effects might be anticipated. The paper then considers a material with regular or periodic heterogeneity, whose behaviour has previously been shown to be consistent with Cosserat elasticity theory, and demonstrates that some of the size effects exhibited by the laminate are also seen in this material. Finally, the size effects predicted for the laminate material are briefly compared to those reported for both virtual and real materials in other literature.

\section{Size effects in a two phase laminated beam}

Figure 1 shows the cross sections of slender rectangular beam samples comprised of a simple laminated material consisting of alternating layers or plies of two different materials of Young's moduli $E_{1}$ and $E_{2}$ respectively. For simplicity all plies of both materials are assumed to be of the same thickness, $t$, and thus all internal layers of the laminate are of the same thickness as illustrated. The central or core layer may be comprised of either of the two materials hence the two variants of the cross section shown in Figure 1. However, the upper and lower surface layers of the sample always consist of the second material with modulus $\mathrm{E}_{2}$ and, in addition, the thickness of these surface layers is always half that of the internal plies. The sample cross section is thus both symmetric, with the neutral axis of bending midway through the section, and comprised of equal proportions of each constituent material. Now if there are $n$ plies of the first material then there will be $n-1$ internal plies together with the two surface plies of the second material so that the cross section is comprised of $2 n+1$ layers in total. The case where $n$ is odd corresponds to the central layer of the beam consisting of the first material while when $n$ is even the central ply will be comprised of the second material. Both cases are illustrated in Figure 1. Bernoulli Euler beam theory allows the flexural rigidity, $D$, of the laminated beam section to be obtained by firstly evaluating the products of the moduli and second moments of area about the section neutral axis of each of the individual plies and then summing these products. When $n$ is odd this summation is represented thus:-

$$
D=2 \int_{0}^{t / 2} E_{1} b y^{2} d y+\sum_{i=1}^{(n-1) / 2} 2 \int_{(i-1 / 2) t+i t}^{(i+1 / 2) t+i t} E_{1} b y^{2} d y+\sum_{i=1}^{(n-1) / 2} 2 \int_{(i-1 / 2) t+(i-1) t}^{(i-1 / 2) t+i t} E_{2} b y^{2} d y+2 \int_{(n / 2) t+[(n-1) / 2] t}^{n t} E_{2} b y^{2} d y
$$

and when $n$ is even the summation becomes:-

$$
D=2 \int_{0}^{t / 2} E_{2} b y^{2} d y+\sum_{i=1}^{n / 2} 2 \int_{(i-1 / 2) t+i t}^{(i+1 / 2) t+i t} E_{1} b y^{2} d y+\sum_{i=1}^{n / 2-1} 2 \int_{i t+(i-1 / 2) t}^{i t+(i+1 / 2) t} E_{2} b y^{2} d y+2 \int_{(n / 2) t+[(n-1) / 2] t}^{n t} E_{2} b y^{2} d y
$$

where $b$ is the breadth of the beam and $y$ the distance from the neutral axis. The core layer is accounted for by the first term in each of these expressions while the summation terms are associated with all remaining internal layers and the final integrals account for the surface 
layers which are always comprised of the second material. Interestingly, when these summations are evaluated the flexural rigidity is given by following single expression:-

$$
D=\frac{E_{1} b n t^{3}}{12}\left[4 n^{2}-3\right]+\frac{E_{2} b n t^{3}}{12}\left[4 n^{2}+3\right]
$$

in both cases.

The depth, $d$, of the beam is:-

$$
d=2 n t
$$

and if the length to depth aspect ratio of the beam is $a$ it follows that the length, $L$, of the beam is given by

$$
L=2 a n t
$$

When loaded in three point bending the stiffness, $K$, of the beam will therefore be:-

$$
K=\frac{E_{1} b n}{2 a^{3} n^{3}}\left[4 n^{2}-3\right]+\frac{E_{2} b n}{2 a^{3} n^{3}}\left[4 n^{2}+3\right]
$$

which can be rearranged thus:-

$$
K=\frac{4\left(E_{1}+E_{2}\right) b}{2 a^{3}}+\frac{3\left(E_{2}-E_{1}\right) b}{2 a^{3} n^{2}}
$$

The motivation for considering three point bending in particular is its practical simplicity which frequently renders it the loading mode of choice; the stiffness of the beam when loaded in other bending configurations could of course be derived similarly.

Now, given that the ply thicknesses of both constituent materials are the same then if the samples were loaded in uniaxial tension parallel to the plies then the material modulus, $E^{*}$, would simply be:-

$$
E^{*}=\frac{E_{1}+E_{2}}{2}
$$

and thus the first term in (7) could be simplified so that the expression for the stiffness of the beam becomes:- 


$$
K=\frac{4 E^{*} b}{a^{3}}+\frac{3\left(E_{2}-E_{1}\right) b}{2 a^{3} n^{2}}
$$

In (9) the first term can be regarded as the stiffness of a slender homogeneous beam of modulus $E^{*}$ loaded in three point bending. The second term however gives rise to a size effect resulting from the heterogeneous nature of the laminated beam. Equation (9) can be compared to the expression for the stiffness of a slender micropolar beam:-

$$
K=4 E^{*} b\left(\frac{d}{L}\right)^{3}\left[1+\left(\frac{l_{c}}{d}\right)^{2}\right]
$$

which was derived by assuming that on every cross section of the beam a linear variation in bending stress and a uniform state of couple stress act [Beveridge et al., 2013] and that across the breadth of the beam any transverse deformations can be ignored. Equation (10) represents a simplification of the more general solution for the deformation quoted previously [Lakes, 1995]. The constitutive parameter, $l_{c}$ that is usually termed the characteristic length, quantifies the length scale associated with the couple stresses. Equation (10) implies that both the modulus and the the characteristic length can be determined from the size effect that may be observed when the stiffness of material samples of differing sizes but with the same aspect ratio, $L / d$, and breadth, $b$, are loaded in three point bending. Experimental testing and detailed finite element analysis of slender beam samples of a heterogeneous material comprised of periodically distributed circular voids within a homogeneous matrix confirmed the validity of equation (10) [Beveridge et al., 2013]. Beam stiffness was found to increase linearly with the reciprocal of beam depth squared, $1 / d^{2}$, in accordance with equation (10). Characteristic length values were determined from the gradient of the stiffness variation while modulus values were identified from the interception of this variation with the stiffness axis. Subsequently, comparable behaviour was observed in slender ring samples with similar heterogeneity that were loaded diametrically [Waseem et al., 2013] and in ring samples in which the topology of the void array constituting the heterogeneity was varied [McGregor \& Wheel, 2014].

Equation (10) predicts that as sample size is reduced there will be a corresponding increase in stiffness; a size effect that agrees with the forecasts of more generalized continuum theories such as micropolar and micromorphic elasticity and with observed behaviour as already noted. However, in the case of the laminate equation (9) implies that size effects may be more elaborate since the second term depends on the relative magnitudes of the ply moduli, $E_{1}$ and $E_{2}$ and thus it may either amplify or modulate the stiffness at any particular sample size.

Figures 2 and 3 show how the predicted stiffness varies with sample size for different combinations of constituent material moduli. The stiffness variations shown in these figures assume an aspect ratio, $a$, of 10 and a common breadth, $b$, of unity. In both figures the sample size is quantified by the reciprocal of depth squared, $1 / d^{2}$, to enable the predicted size effects 
to be compared directly with the forecasts of equation 10. Furthermore, this size measure, $1 / d^{2}$, has been normalized with respect to that of the thinnest possible beam, this being $1 /(2 t)^{2}$ while the stiffness has been normalized with respect to that of a beam of almost infinite depth, that is, a beam for which $\mathrm{n}$ is very large implying that the second term in equation (9) diminishes to zero and any size effect becomes insignificant.

Figure 2 shows the size effects predicted by equation (9) when one of the materials is 10 times stiffer than the other. Evidently, when the material constituting the surface layers is the stiffer of the two, that is $\mathrm{E}_{1}=0.1 \mathrm{E}_{2}$, a positive size effect in which stiffness increases as size reduces is predicted. Furthermore, this positive size effect is apparently linear as anticipated by equation 10 . However, when $\mathrm{E}_{1}=10 \mathrm{E}_{2}$ and the surface is therefore formed from the more compliant material a contrasting, negative size effect is seen. Nevertheless, this negative effect appears to vary linearly in accordance with its positive counterpart. Interestingly, although these size effects are entirely different in nature the magnitude of the rate at which the stiffness varies appears to be similar in both cases.

Figure 3 shows the corresponding stiffness variations when the ratio of the material moduli is increased by a further factor of 10 . When $\mathrm{E}_{1}=0.01 \mathrm{E}_{2}$ implying the surfaces are comprised of the stiffer material, a positive size effect is again seen while when $E_{1}=100 \mathrm{E}_{2}$ and the surfaces are more compliant a negative size effect results once more. It is also interesting to note that when presented in this non dimensional manner both of the size effects seen at this ratio of material moduli reflect those shown in Figure 2. However, when equation (10) is used to determine characteristic length data from the positive size effects depicted in the two figures then a value of $1.57 t$ for $l_{c}$ is obtained when $\mathrm{E}_{1}=0.1 \mathrm{E}_{2}$ but increases to $1.72 t$ when the ratio of moduli is changed such that $\mathrm{E}_{1}=0.01 \mathrm{E}_{2}$.

\section{Size effects in a two dimensional material with periodic heterogeneity}

Figure 4 illustrates a material with regular heterogeneity created by introducing a periodic array of circular voids into an otherwise classically elastic matrix material. The constitutive behaviour of materials of this type has been investigated previously [Beveridge et al., 2013] in the context of generalized continua and the behaviour shown to be consistent with the predictions of micropolar elasticity theory. Prescribing the void radius, $\mathrm{V}_{\mathrm{R}}$, together with the separation of the void centres, $S_{x}$ and $S_{y}$, in the indicated $x$ and $y$ directions respectively is sufficient to fully define the geometry of the heterogeneity. The void centres thus lie on a triangular grid. When $S_{y}=\sqrt{ } 3 S_{x} / 2$ a detailed finite element analysis of a representative piece of material incorporating a sufficiently large number of voids revealed that the material exhibited approximate planar isotropy. Thus the material is transversely isotropic. The analysis assumes that the matrix material exhibits classically elastic behaviour as quantified by its Young's modulus and Poisson's ratio.

This material was shown to exhibit a size dependent stiffening consistent with equation 10 through finite element analysis of slender beam samples of different depths but the same aspect ratio [Beveridge et al., 2013]. In producing the mesh required to represent each beam 
sample the geometric details of the heterogeneity were explicitly incorporated by firstly paving the rectangular region around a particular void with the structured array of quadratic quadrilateral elements illustrated in figure 5 and then repeatedly regenerating this array of elements at suitable spatial increments to represent an entire sample. One consequence of generating the entire sample representation in this way was that the upper and lower sample surfaces were each located midway between adjacent rows of voids as illustrated in figure 6 and thus these surfaces intersected none of the voids. However, this is not the only manner in which a finite sized beam sample could be identified from within an infinite sheet of the perforated material. A sample could just as easily be identified in alternative ways and in some of these the surfaces may intersect the voids. Figure 6 shows one such alternative in which the structured mesh shown in figure 7 was employed to represent the region of matrix material located between a specific void and its neighbours. Consequently, when this mesh is repeatedly regenerated to represent an entire sample the sample surfaces periodically bisect all voids in a given row as shown in figure 6 . Both means of mesh generation were therefore used to analyse beams of increasing depth, this being determined by the number of rows of voids as shown in figure 6 . The length to depth aspect ratio was set at 10.4:1 for all beams while the void separations, $S_{x}$ and $S_{y}$, were prescribed at $1.0 \mathrm{~mm}$ and $0.866 \mathrm{~mm}$ respectively thus fixing the overall dimensions of each beam. For the matrix material plane stress behaviour was assumed while Young's modulus and Poisson's ratio were set to $20 \mathrm{GPa}$ and 0.3 respectively. Constraints and loading representative of three point bending were applied. However, to reduce computational effort suitable boundary conditions were imposed at the central loading plane to exploit the symmetries in geometry and loading and thereby facilitate analysis of only one half of each beam.

Figure 8 shows the predicted variations in beam stiffness with size for different void volume fractions, Vf, when the sample surfaces do not intersect the array of voids. The stiffness variation appears to be approximately linear at any given void size. The characteristic length of each material can be obtained from the gradient of the corresponding variation while the modulus of can be derived from the intercept according to equation (10). Table 1 lists values of each of these constitutive parameters as a function of void radius and volume fraction. This table also lists data derived from the stiffness variations determined for more slender samples with an increased aspect ratio of 20.8:1. These data imply that as void radius increases the material modulus decreases, as might be expected since there is less material to support the applied loading, while the characteristic length increases. Furthermore, the values of this latter parameter obtained at aspect ratios of 10.4:1 and 20.8:1 only vary slightly implying that although slender beam behaviour is assumed in equation (10) the lower, 10.4:1, aspect ratio beams are sufficiently slender enough to provide very reasonable estimates of the characteristic length since they appear to satisfy this underlying assumption. Figure 9 shows the relationship between void radius and characteristic length for the higher, 20.8:1, aspect ratio beams. This relationship is evidently linear as anticipated by a previous theoretical prediction [Bigoni \& Drugan, 2007].

Figure 10 shows variations in beam stiffness with size for the same void radii when the aspect ratio is set at $10.4: 1$ but the surfaces now bisect the voids. These variations each show a 
decrease in stiffness with reducing size and therefore no longer concur with equation (10). However, each variation is nonetheless linear which does accord with equation 9 for the case where the sample surfaces are comprised of the more compliant material. Additionally, the intercept of a given negative size effect seen in figure 10 corresponds to that obtained from the positive effect shown in figure 8 for the equivalent void radius. This correspondence thus reflects the convergence of both size effects seen in the laminate material at large beam depths and illustrated in figure 2. Figure 11 shows how the magnitudes of both the positive size effects seen in figure 8 and their negative counterparts shown in figure 10 vary with void volume fraction. This figure suggests that the magnitude of the negative effect broadly reflects that of the positive effect. Moreover, when the void volume fraction is low then both size effects are small since the beam samples are predominantly comprised of matrix material. The magnitudes of each effect then increase as void volume fraction increases. However, both magnitudes reach a maximum beyond which they then diminish as the samples become predominantly comprised of voids.

\section{Discussion and conclusions}

Size effects forecasts were made previously [Tekoglu \& Onck, 2008] for virtual two dimensional foams. The stochastic cellular microstructure was represented as random Voronoi tessellations with Timoshenko beam finite elements being used to represent individual cell wall sections. Closed polygons were used to represent all internal cells within an elongated rectangular region. However, for those cells intersecting the boundary no elements were located coincident to the boundary to facilitate their closure and they remained open. The finite element representations were loaded in pure bending of the major axis and multiple analyses were conducted using a different randomly generated finite element mesh on each occasion in order to capture the behaviour of the intrinsically stochastic microstructure. Bending stiffness was found to vary with beam depth which was altered by changing the lesser dimension of the representative rectangular region. At small depths forecast stiffness was less than that anticipated by classical elasticity theory. However, as depth increased stiffness was predicted to rise and asymptotically approach the classical result. This behaviour appears to arise from the rupture of those cells adjacent to the boundaries which results in a local increase in compliance thereby compromising bending stiffness and ultimately resulting in a negative size effect rather than a positive one that might be expected for a generalized continuum. Thus the negative size effect forecast for the stochastic foam reflects that seen in both the present laminate and voided materials when stiffness of the material adjacent to the surface is deliberately compromised.

Contradictory size effects have been reported previously in human cortical bone [Yang \& Lakes, 1982; Choi et al., 1990] where samples were loaded in bending experiments in each case. While the earlier work reported a positive size effect the later reported an opposite, negative effect. In the earlier work beam specimens with depths down to $1.4 \mathrm{~mm}$ were tested but in the later samples with substantially smaller depths were also investigated. While a mild positive size effect was reported earlier the most significant effect was observed in the small samples investigated in the later work and, moreover, this effect was unequivocally negative. 
The investigators suggested a qualitative explanation of this size effect based on the exposure of the major microstructural feature, the vascular channel or Haversian canal system, at the sample surfaces giving rise to an increase in compliance of the material located in the vicinity. However, the effect was interpreted quantitatively as a reduction in the apparent modulus of the material rather than the behaviour of a more generalized continuum. Nevertheless, the negative effect reported for cortical bone appears to reflect that seen in the laminate and voided materials considered here.

These negative size effects seen in both stochastic foams and cortical bone have not been interpreted in the context of generalized continuum theories. Presumably since these effects appear to contradict the predictions of such theories identification of relevant constitutive properties was not attempted. However, constitutive property identification is paramount since it provides a rational basis for comparing the practical performance of materials when loaded. Apparently this cannot be realized in the case of a heterogeneous material that displays a negative size effect. Nevertheless, the correspondence between the positive and negative size effects forecast for both the simple laminated and the more involved perforated materials considered suggest a pragmatic, albeit empirical, resolution to this dilemma since the correspondence suggests that it may be possible to infer constitutive property data from observed effects in cases where these are negative. 


\section{References}

Anderson, W.B. and Lakes, R.S. (1994), Size effects due to Cosserat elasticity and surface damage in closed-cell polymethacrylimide foam. Journal of Materials Science, 29(24):64136419

Beveridge, A.J., Wheel, M.A. \& Nash, D.H. (2013), The Micropolar Elastic Behaviour of Model Macroscopically Heterogeneous Materials, International Journal of Solids \& Structures, 50, 246-255

Bigoni, D. and Drugan, W.J. (2007), Analytical derivation of Cosserat moduli via homogenization of heterogeneous elastic materials, Journal of Applied Mechanics, 74, 741753

Choi, K., Kuhn, J.L.,Ciarelli, M.J. and Goldstein, S.A. (1990), The elastic moduli of human subchondral, trabecular, and cortical bone tissue and the size-dependency of cortical bone modulus. Journal of Biomechanics, 23(11), 1103-1113.

Eringen, A.C., (1999), Microcontinuum Field Theories I: Foundations and Solids. SpringerVerlag New York

Forest, S. and Sab, K., (1998), Cosserat overall modeling of heterogeneous materials, Mech. Res. Comm., 25 (4), pp. 449-454

Gao, X.L. and Mahmoud, F.F., (2014), A new Bernoulli-Euler beam model incorporating microstructure and surface energy effects, Zeitschrift fur angewandte Mathematik und Physik (ZAMP), 65, 393-404

Herrmann, G. and Achenbach, J.D., (1968), Applications of theories of generalized Cosserat continua to the dynamics of composite materials. In: Kröner, E. (Ed.), Mechanics of Generalized Continua, Proc. IUTAM Symposium, 69-79, Springer, Berlin

Lakes, R.S., (1983), Size effects and micromechanics of a porous solid. Journal of Materials Science, 18, 2572-2580

Lakes, R.S., (1986), Experimental microelasticity of two porous solids. International Journal of Solids and Structures, 22, 55-63

Lakes, R.S., (1995), Experimental methods for study of Cosserat elastic solids and other generalized elastic continua. in Continuum models for materials with micro-structure (Ed. Mühlhaus H.), Wiley, New York, 1-25

McGregor, M. and Wheel M.A., (2014), On the coupling number and characteristic length of micropolar media of differing topology, Proceedings of the Royal Society A, 470

Tekoglu, C. and Onck, P.R. (2008), Size effects in two-dimensional Voronoi foams: A comparison between generalized continua and discrete models, Journal of the Mechanics and Physics of Solids, 56, 3541-3564 
Waseem, A., Beveridge, A. J., Wheel, M.A. and Nash, D. (2013), The influence of void size on the micropolar constitutive properties of model heterogeneous materials, European Journal of Mechanics A: Solids. 40, 148-157

Yang, J.F.C. and Lakes, R.S., (1982), Experimental study of micropolar and couple stress elasticity in bone in bending. Journal of Biomechanics, 15, 91-98 


\begin{tabular}{|c|c|c|c|c|c|c|}
\hline \multirow{2}{*}{$\begin{array}{c}\text { Void } \\
\text { Diameter, } \\
\mathrm{V}_{\mathrm{d}}(\mathrm{m})\end{array}$} & \multirow{2}{*}{$\begin{array}{c}\text { Void } \\
\text { Fraction } \\
\text { Vf }\end{array}$} & \multirow{2}{*}{$\begin{array}{c}\text { Normalised } \\
\text { void radius } \\
\mathrm{V}_{\mathrm{R}} / \mathrm{S}_{\mathrm{Y}}\end{array}$} & \multicolumn{2}{|c|}{$\begin{array}{l}\text { Young's Modulus } \\
\text { (GPa) }\end{array}$} & \multicolumn{2}{|c|}{$\begin{array}{l}\text { Characteristic length } \\
(\mathrm{mm})\end{array}$} \\
\hline & & & $\begin{array}{c}10.4: 1 \\
\text { aspect } \\
\text { ratio }\end{array}$ & $\begin{array}{c}20.8: 1 \\
\text { aspect } \\
\text { ratio }\end{array}$ & $\begin{array}{c}10.4: 1 \\
\text { aspect ratio }\end{array}$ & $\begin{array}{c}20.8: 1 \\
\text { aspect ratio }\end{array}$ \\
\hline 0.2 & 0.036 & 0.12 & 17.47 & 17.87 & 0.28 & 0.28 \\
\hline 0.3 & 0.082 & 0.17 & 15.37 & 15.71 & 0.42 & 0.43 \\
\hline 0.4 & 0.145 & 0.23 & 12.90 & 13.16 & 0.55 & 0.57 \\
\hline 0.5 & 0.227 & 0.29 & 10.31 & 10.50 & 0.66 & 0.70 \\
\hline 0.6 & 0.326 & 0.35 & 7.74 & 7.83 & 0.75 & 0.82 \\
\hline
\end{tabular}

Table 1 Comparison of the characteristic lengths for different void radii at 10.4:1 and 20.8:1 length to depth aspect ratios. 


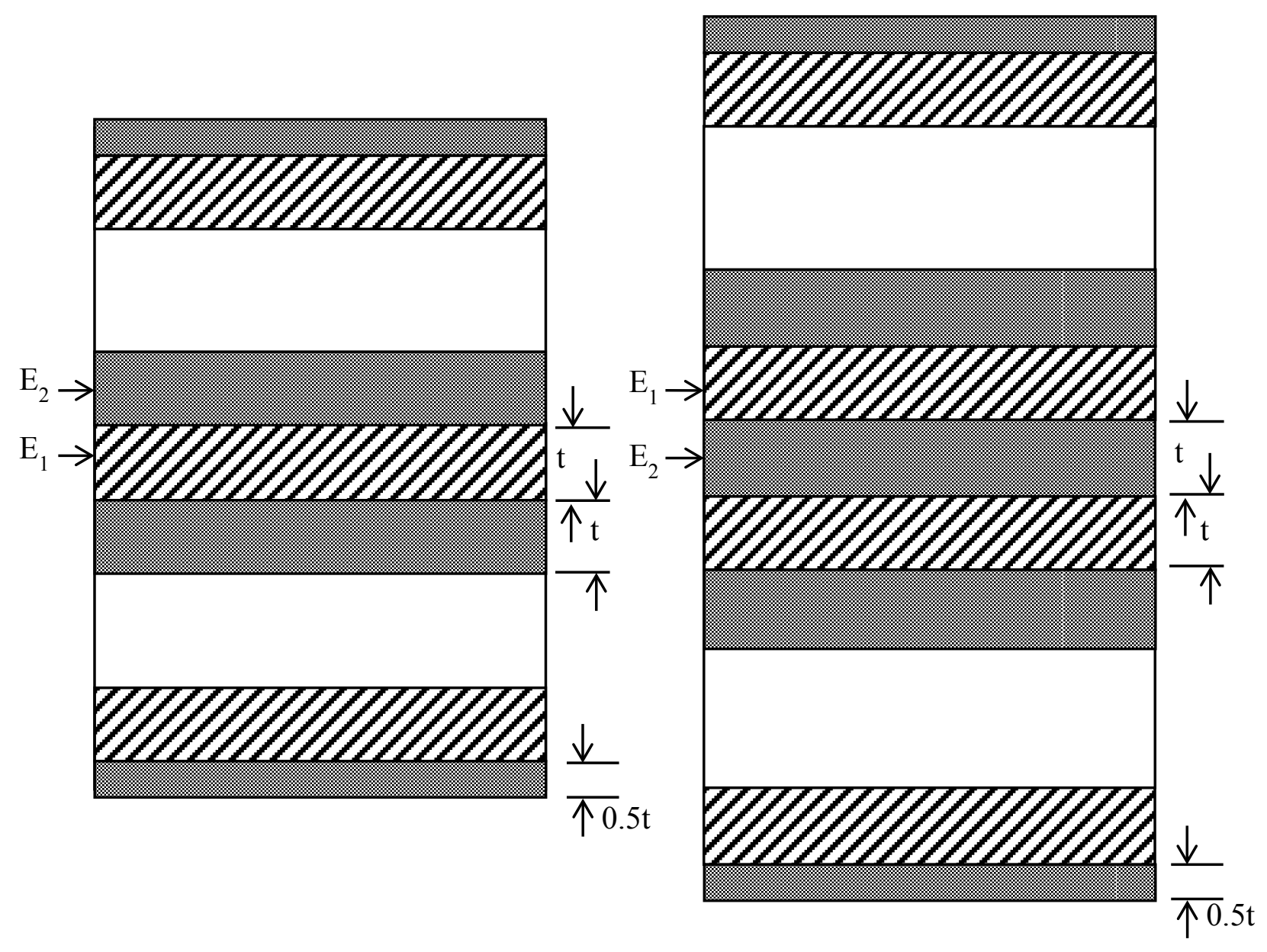

Figure 1 Laminated Beam Model of Heterogeneous Material 


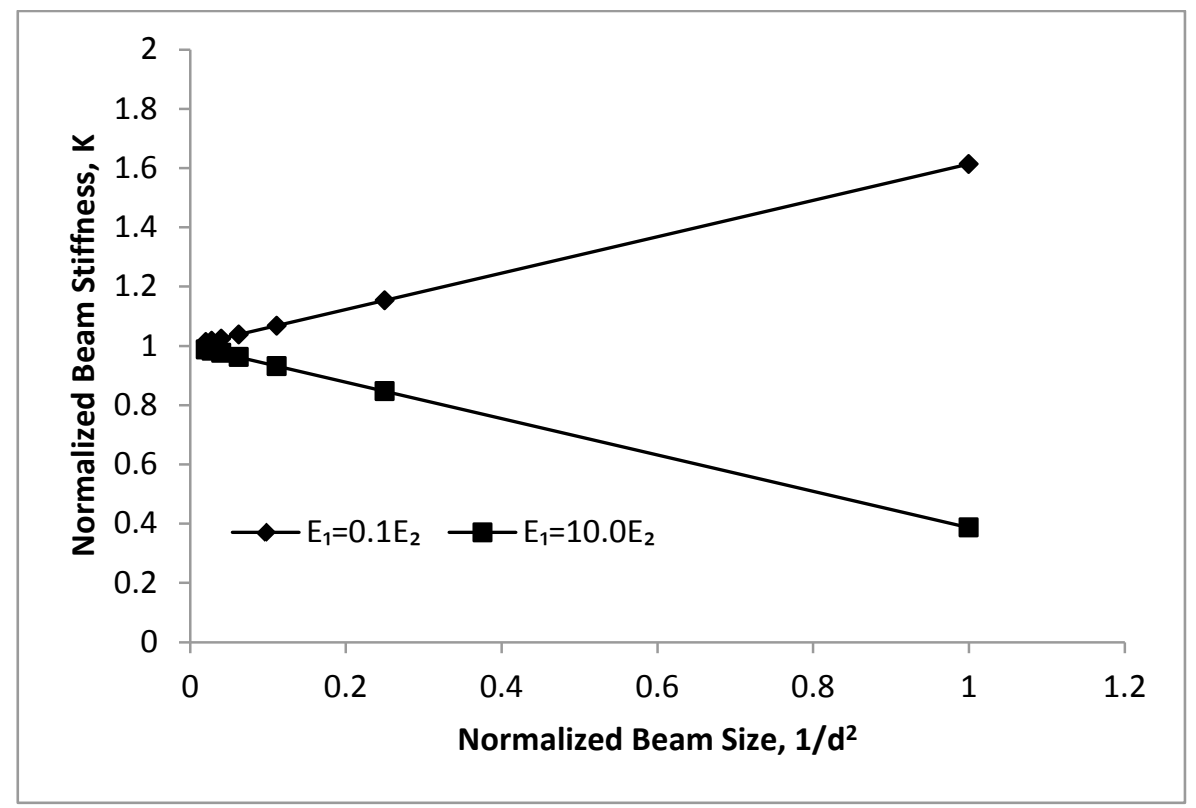

Figure 2 Variation in stiffness with beam size for cases where $\mathrm{E}_{1}=0.1 \mathrm{E}_{2}$ and $\mathrm{E}_{1}=10.0 \mathrm{E}_{2}$ 


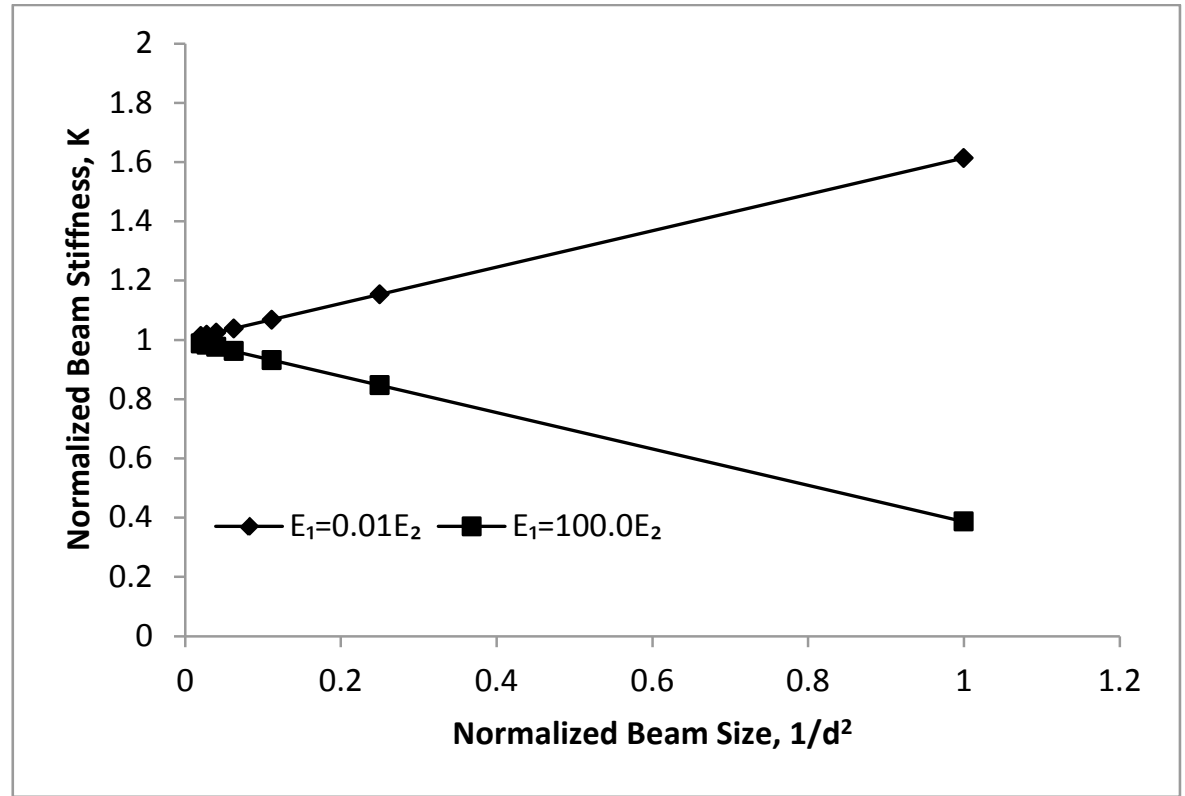

Figure 3 Variation in stiffness with beam size for cases where $\mathrm{E}_{1}=0.01 \mathrm{E}_{2}$, and $\mathrm{E}_{1}=100.0 \mathrm{E}_{2}$ 


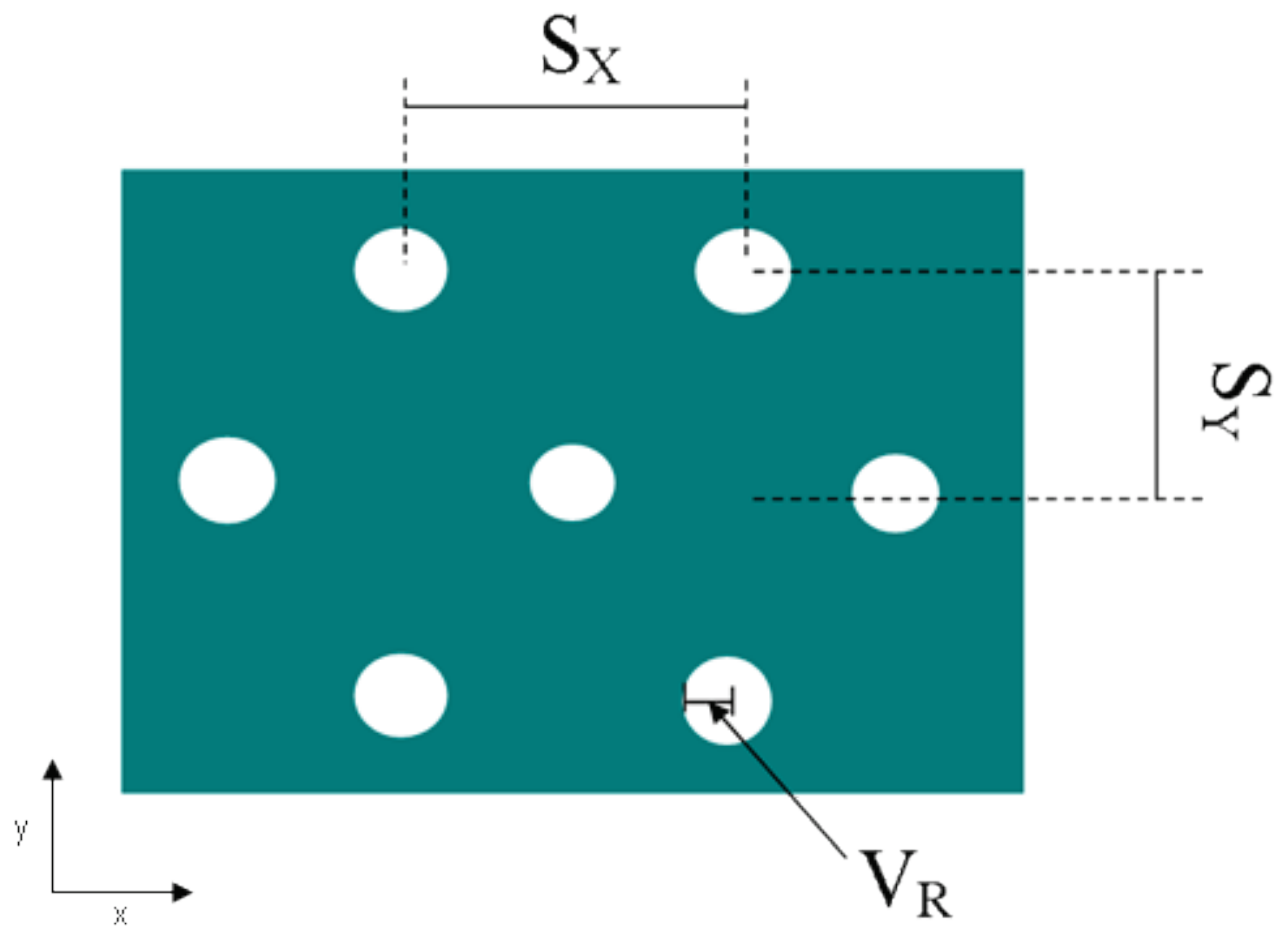

Figure 4 Two dimensional material with regular, periodic heterogeneity investigated previously within the context of micropolar elasticity theory. 


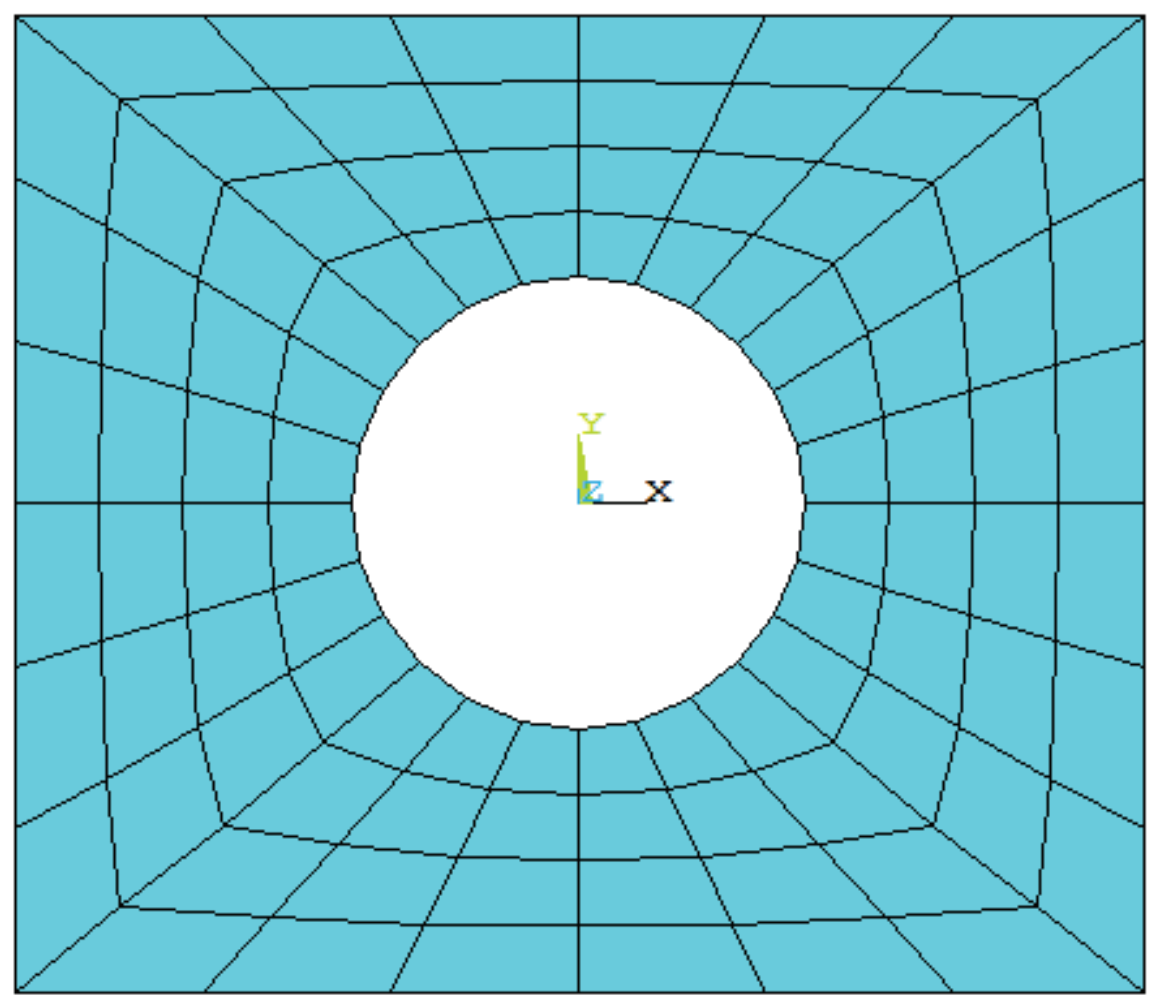

Figure 5 Structured mesh of quadratic quadrilateral finite elements used to represent rectangular region around a particular void within two dimensional heterogeneous material 
nnen

coepecepes

000000000009

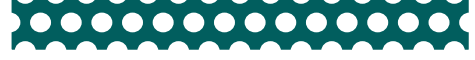

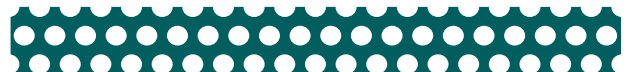

0000000000000000
00000

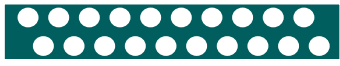

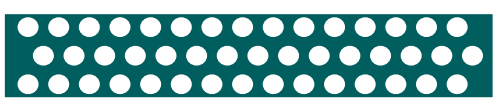

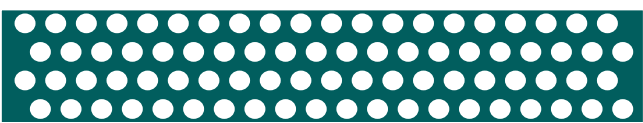

Figure 6 Representation of beam samples of increasing size generated by finite element meshes shown in figures 5 (right) and 7 (left). 


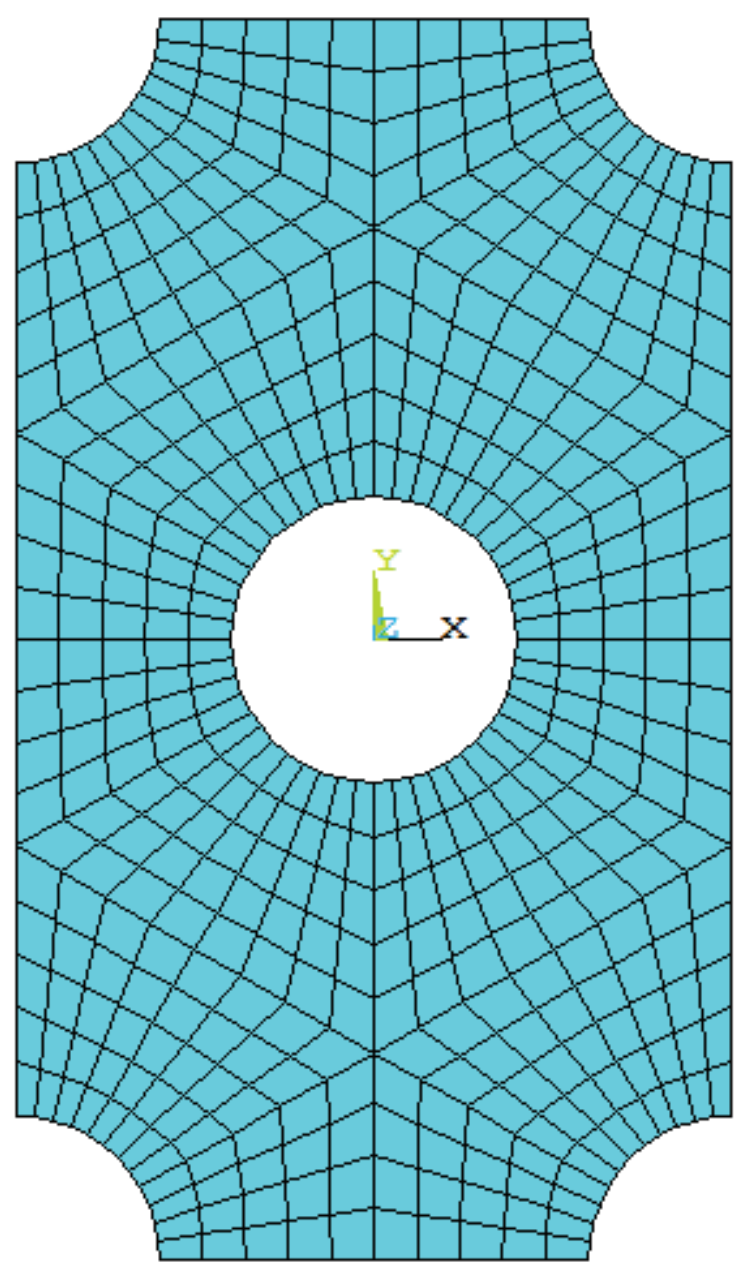

Figure 7 Alternative mesh of quadratic quadrilateral finite elements used to represent region between neighbouring voids within two dimensional heterogeneous material. 


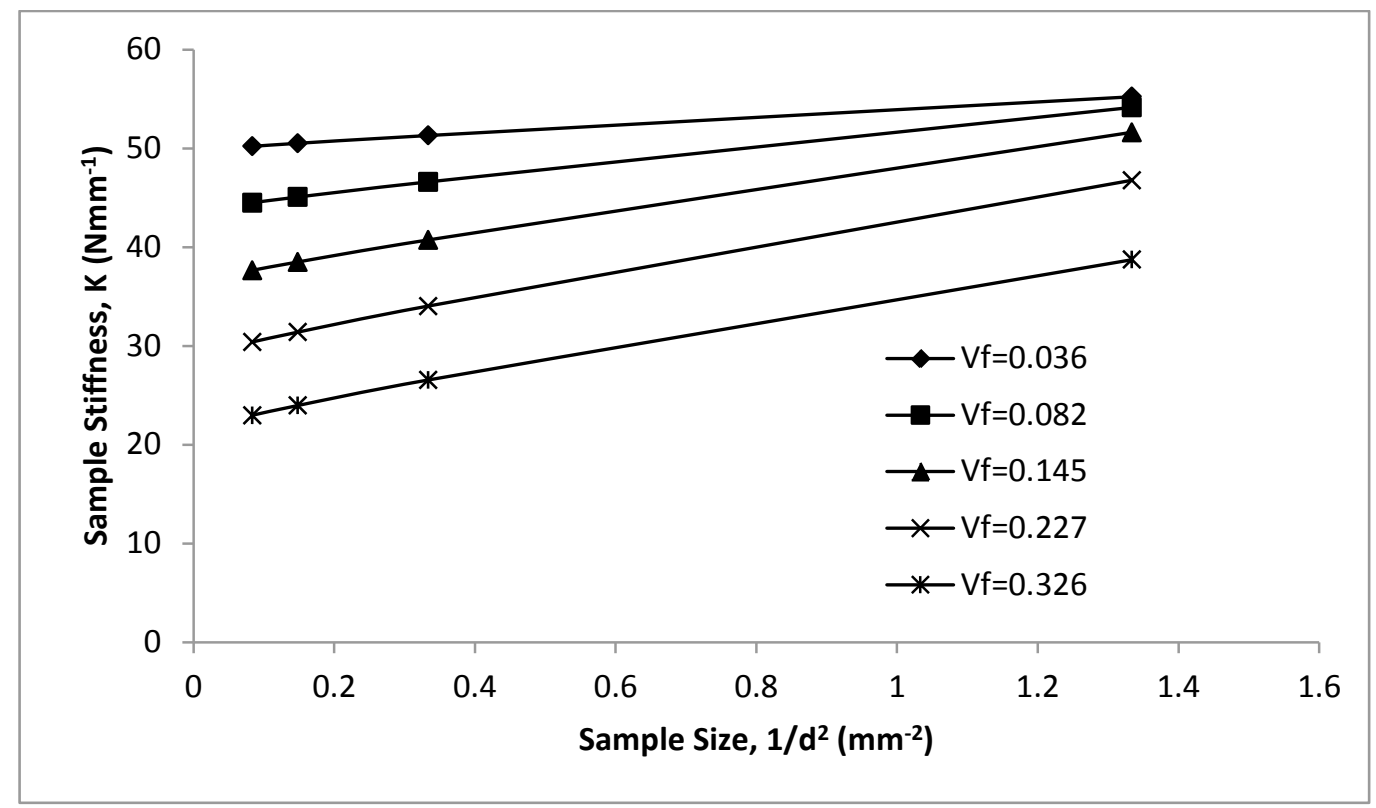

Figure 8 Stiffness against the reciprocal of depth squared for beams with smooth surfaces at a 10.4:1 length to depth aspect ratio for various void volume fractions, Vf, . 


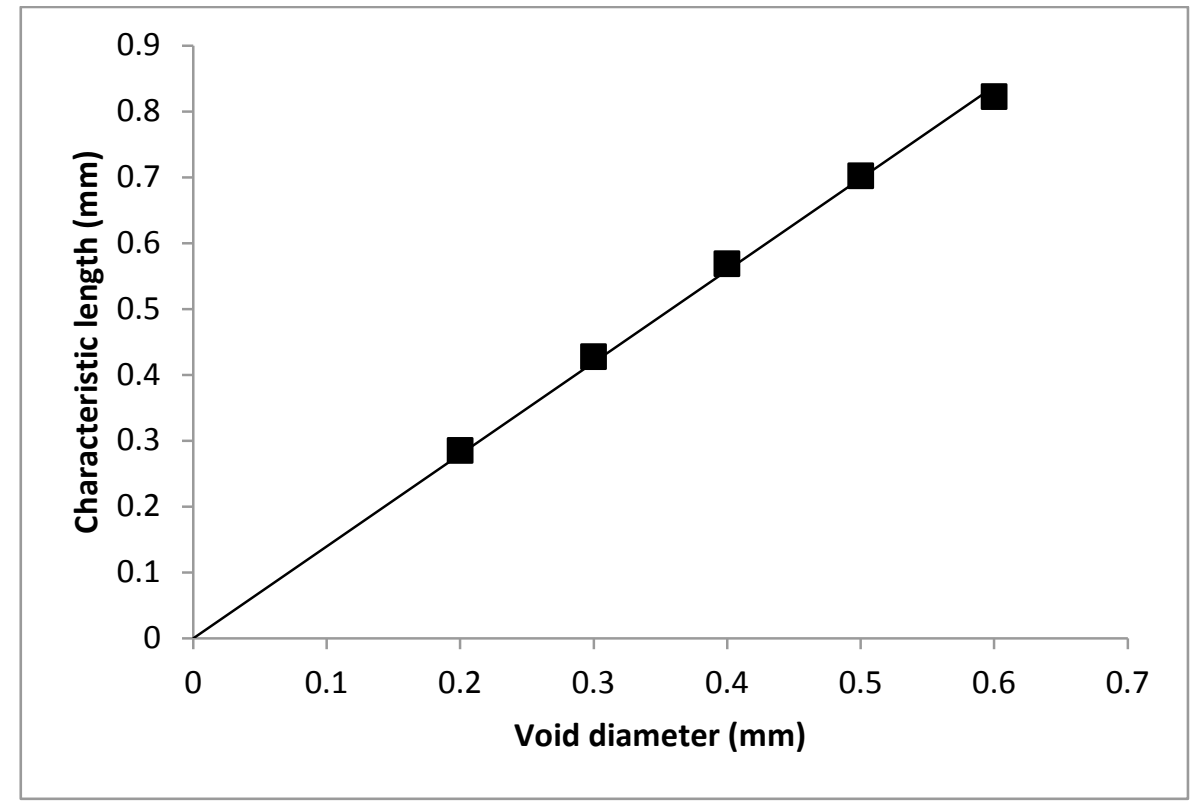

Figure 9 Variation in micropolar characteristic length with void diameter for 20.8:1 aspect ratio beams 


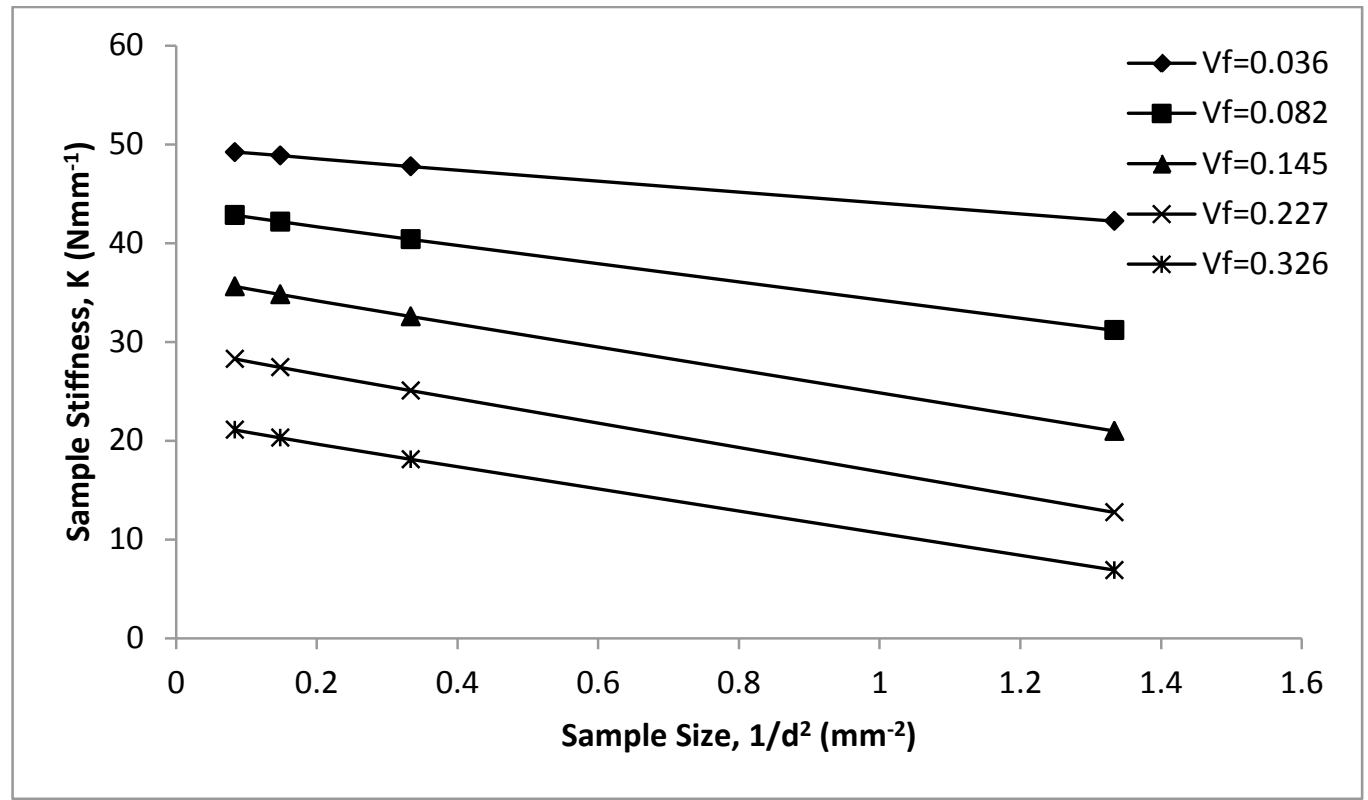

Figure 10 - Stiffness against the reciprocal of depth squared for beams with intersected surfaces at a 10.4:1 length to depth aspect ratio for various void volume fractions, Vf. 


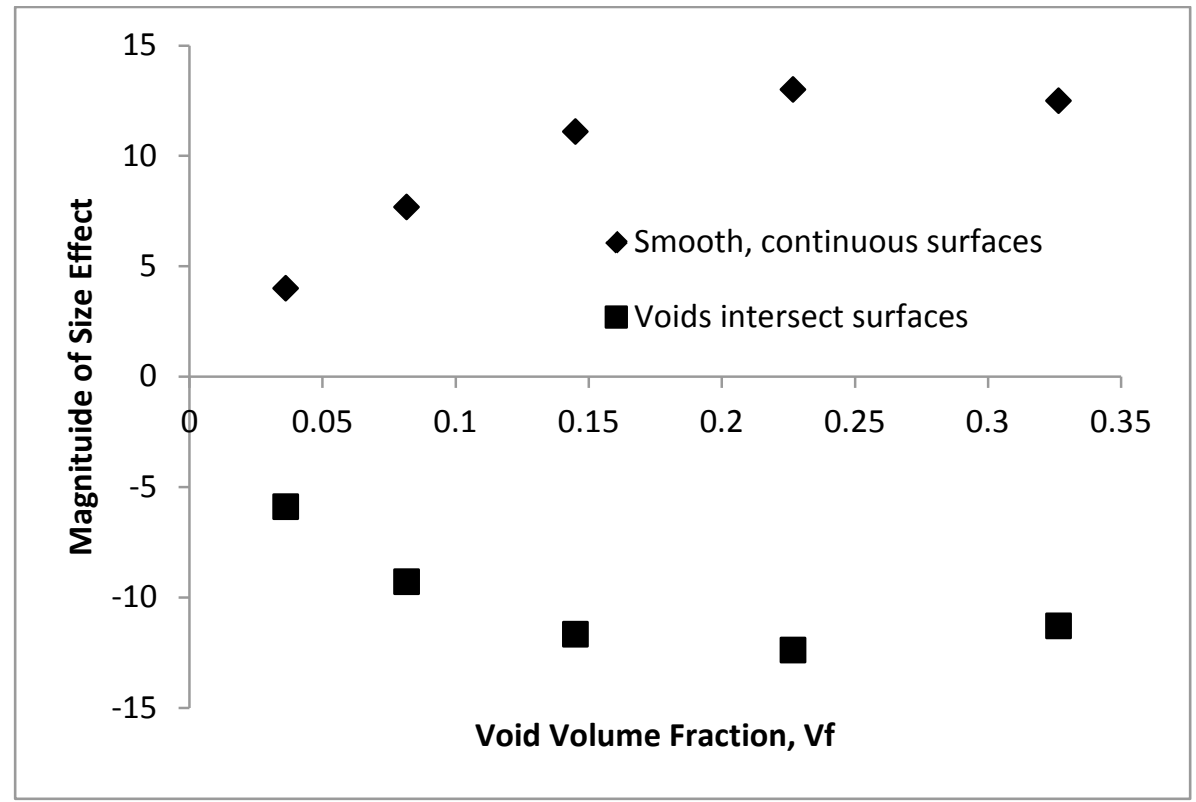

Figure 11 - The magnitude of the size effects as a function of void volume fraction $\mathrm{Vf}$ for beams with both smooth and intersected surfaces and a 20.8:1 length to depth aspect ratio. 